\title{
Mn Impurity in Bulk GaAs Crystals
}

\author{
M. Pawıowski ${ }^{a, b, *}$, M. Piersa ${ }^{a}$, A. Woloś $^{b}$, M. PalczeWska $^{a}$,

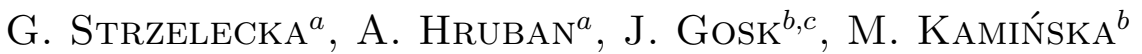 \\ AND A. TWARDOWSKI ${ }^{b}$ \\ ${ }^{a}$ Institute of Electronic Materials Technology \\ Wólczyńska 133, 01-919 Warszawa, Poland \\ ${ }^{b}$ Institute of Experimental Physics, Warsaw University \\ Hoża 69, 00-681 Warszawa, Poland \\ ${ }^{c}$ Faculty of Physics, Warsaw University of Technology \\ Koszykowa 75, 00-662 Warszawa, Poland
}

\begin{abstract}
Magnetic and electron transport properties of GaAs:Mn crystals grown by Czochralski method were studied. Electron spin resonance showed the presence of $\mathrm{Mn}$ acceptor $\mathrm{A}$ in two charge states: singly ionized $\mathrm{A}^{-}$in the form of $\mathrm{Mn}^{2+}\left(d^{5}\right)$, and neutral $\mathrm{A}^{0}$ in the form of $\mathrm{Mn}^{2+}\left(d^{5}\right)$ plus a bound hole (h). It was possible to determine the relative concentration of both types of centers from intensity of the corresponding electron spin resonance lines. Magnetization measured as a function of magnetic field (up to $6 \mathrm{~T}$ ) in the temperature range of $2-300 \mathrm{~K}$ revealed overall paramagnetic behavior of the samples. Effective spin was found to be about 1.5 value, which was consistent with the presence of two types of Mn configurations. In most of the studied samples the dominance of $\mathrm{Mn}^{2+}\left(d^{5}\right)+\mathrm{h}$ configuration was established and it increased after annealing of native donors. The total value of $\mathrm{Mn}$ content was obtained from fitting of magnetization curves with the use of parameters obtained from electron spin resonance. In electron transport, two mechanisms of conductivity were observed: valence band transport dominated above $70 \mathrm{~K}$, and hopping conductivity within Mn impurity band at lower temperatures. From the analysis of the hopping conductivity and using the obtained values of the total $\mathrm{Mn}$ content, the effective radius of $\mathrm{Mn}$ acceptor in GaAs was estimated as $a=11 \pm 3 \AA$.
\end{abstract}

PACS numbers: 71.55.Eq, 72.20.Ee, 75.50.Pp, 76.30.Fc

\section{Introduction}

Diluted magnetic semiconductors based on III-Mn- $\mathrm{V}$ compounds have been a subject of intense studies over recent years not only due to their interesting phys-

*corresponding author; e-mail: mpawl@itme.edu.pl 
ical properties, but also due to the possibility of application in spin-electronic devices. Ferromagnetic behavior observed in some of them has been attributed to localized high spins of substitutional Mn centers in $d^{5}$ configuration and exchange interactions between such spins by means of free holes (RKKY-type coupling between Mn ions). Concentrations of both species: Mn centers and free holes have been found to directly influence the value of Curie temperature $T_{\mathrm{C}}$. In GaMnAs, which possesses the record high $T_{\mathrm{C}}$ (above $170 \mathrm{~K}$ ), most Mn atoms substitute for three-valent cations of GaAs host, and act as ionized acceptors leading to creation of holes in the valence band. Infrared optical experiments have indicated that neutral Mn acceptor center in GaAs is of a type of $\left[\mathrm{Mn}^{2+}\left(d^{5}\right)+\right.$ a weakly bound hole (h) [1]. However, the localization radius of such hole has not been determined up to now.

Since ferromagnetic properties of III-Mn-V semiconductors are connected with Mn configuration, it is essential to understand the behavior of Mn centers. Especially important seems to be parameters describing Coulombically bound holes, since one of the possible exchange mechanisms could involve such holes.

In this paper we report results of our studies performed on GaAs:Mn in order to better understand properties of Mn centers in this compound.

\section{Experimental}

GaAs:Mn crystals were grown by Czochralski method. Manganese was added during growth and its concentration $n_{\mathrm{Mn}}$ was varied in the range of $10^{16}$ to $10^{19} \mathrm{~cm}^{-3}$ by changing growth conditions (see the Table). Electron spin resonance (ESR) experiments were carried out using a Bruker ESP-300 X-band spectrometer with $100 \mathrm{kHz}$ field modulation and phase-sensitive detection, which operated at a microwave frequency of about 9.4 GHz. A helium gas-flow Oxford Instruments cryostat was used for variable temperature measurements, starting from $6 \mathrm{~K}$. Magnetization of the samples was studied as a function of magnetic field (up to $6 \mathrm{~T}$ ) and in the temperature range of 2 to $300 \mathrm{~K}$, using a superconducting quantum interference device (SQUID). Hall measurements were performed in the standard of Van der Pauw configuration under weak magnetic field (below $0.5 \mathrm{~T}$ ) in the temperature range from 7 to $320 \mathrm{~K}$.

\section{Results and discussion}

Representative ESR spectra of the investigated samples are shown in Fig. 1a. They revealed the presence of two groups of lines. The first one, detected at magnetic field around 170 and $330 \mathrm{mT}$ has been assigned to the absorption of singly ionized $\mathrm{A}^{-}\left(\mathrm{Mn}^{2+}\left(d^{5}\right)\right)[2]$. The second one, observed only at low temperatures and at magnetic field around 110 and $230 \mathrm{mT}$ has been connected with the absorption of neutral $\mathrm{A}^{0}\left(\mathrm{Mn}^{2+}\left(d^{5}\right)+\mathrm{h}\right)$ configuration [3]. For both types of centers it was possible to observe the allowed $(\Delta M=1)$ and forbidden $(\Delta M=2)$ transitions (see Fig. 1). The observation of forbidden transitions was an indication of Mn impurity- 


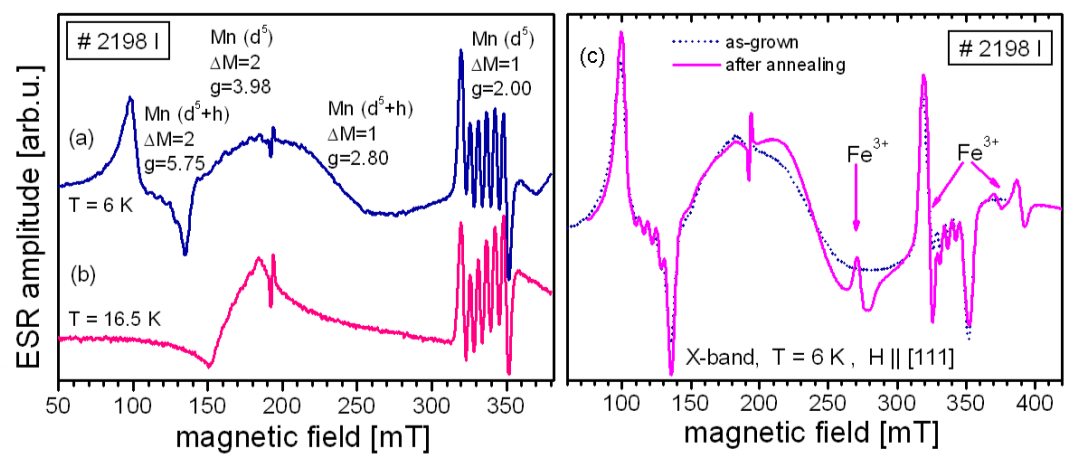

Fig. 1. ESR spectra of $p$-type GaAs:Mn sample \# 2198 I with signals corresponding to neutral $\mathrm{A}^{0}$ and ionized $\mathrm{A}^{-} \mathrm{Mn}$ acceptor, measured at $6 \mathrm{~K}$ (a) and $16.5 \mathrm{~K}$ (b). (c) ESR spectra of sample \# 2198 I for as-grown sample (dot line) and after annealing at $950^{\circ} \mathrm{C}$ (solid line).

-induced crystal disorder or was a consequence of lack of zero-field splitting. The corresponding hyperfine interaction constant $A$ and $g$-factors for $\mathrm{Mn}^{2+}\left(d^{5}\right)$ were the following: $A=5.6 \mathrm{mT}, g=2.000 \pm 0.003(\Delta M=1)$ and $g=3.96 \pm 0.03$ $(\Delta M=2)$, whereas for $\mathrm{Mn}^{2+}\left(d^{5}\right)+\mathrm{h}: A=6.0 \mathrm{mT}, g=2.80 \pm 0.03(\Delta M=1)$ and $g=5.75 \pm 0.03(\Delta M=2)$. Increasing temperature caused a decrease in the $\mathrm{A}^{0}$ line intensities and the lines disappeared completely around $13 \mathrm{~K}$ (see Fig. 1b). On the other hand, ESR lines attributed to $\mathrm{A}^{-}$were still visible even at temperatures above $60 \mathrm{~K}$.

The areas under ESR lines of $\mathrm{A}^{-}$were similar for all samples with intermediate $\mathrm{Mn}$ concentrations (hole concentration $p$ of the order of $10^{17} \mathrm{~cm}^{-3}$ at room temperature (RT)). Presumably $\mathrm{Mn}$ created a singly ionized acceptor $\mathrm{A}^{-}$when it compensated residual donor impurities (S, Se) or native donor defects (i.e. donors arising during post growth crystal cooling, such as As vacancies and their complexes with Ga vacancies or with arsenic antisities [4]). Thermal annealing $\left(950^{\circ} \mathrm{C}\right.$, $2 \mathrm{~h}$, As atmosphere) caused annihilation of native donor defects and $\mathrm{A}^{0} \mathrm{ESR}$ lines became narrower (especially $\Delta M=1$ line) and more intense (see Fig. 1c). Simultaneously, singly ionized acceptors $\mathrm{Fe}^{2+}\left(d^{6}\right.$, no ESR absorption) changed their charge state to $\mathrm{Fe}^{3+}\left(d^{5}\right)$ and their resonance lines appeared. The change in intensity of $\mathrm{A}^{-}$-related resonances was not clearly seen since the lines were overlapped by the Fe lines (see Fig. 1c). For samples with high Mn concentrations ( $p$ equal $9 \times 10^{17}$ or $3 \times 10^{18} \mathrm{~cm}^{-3}$ at RT) additional lines with axial [111] symmetry, arising from a complex of $\mathrm{Mn}^{2+}$-donor impurities [5] were observed. A part of these lines was in the same range of magnetic field as $\mathrm{A}^{-}$lines and, therefore, it was not possible to estimate the area under $\mathrm{Mn}^{2+}\left(d^{5}\right)$ signal. For the same two samples, the lines related to $\mathrm{Mn}^{2+}\left(d^{5}\right)+\mathrm{h}$ became very broad, the hyperfine structure of $\Delta M=2$ transitions completely disappeared and the area under $\mathrm{A}^{0}$ lines could be only roughly evaluated. 
TABLE

Hall concentrations measured at RT, the ratios of the areas under ESR $\mathrm{A}^{0}$ lines to $\mathrm{A}^{-}$lines and total Mn concentrations obtained from fits to the magnetization experimental data of GaAs:Mn samples.

\begin{tabular}{c|c|c|c|c}
\hline \hline Sample & \multirow{2}{*}{$\begin{array}{c}p \text { at RT } \\
{\left[\mathrm{cm}^{-3}\right]}\end{array}$} & $\begin{array}{c}\text { Ratio of } \\
\left(d^{5}+\mathrm{h}\right) / d^{5}\end{array}$ & \multicolumn{2}{|c}{ Calculated $n_{\mathrm{Mn}}$} \\
\cline { 4 - 5 } & {$[\%$ at. $]$} & {$\left[\mathrm{cm}^{-3}\right]$} \\
\hline $2200 \mathrm{I}$ & $3.0 \times 10^{16}$ & 0.8 & & $>3.0 \times 10^{16}$ \\
$2200 \mathrm{II}$ & $2.8 \times 10^{17}$ & 2.9 & 0.0031 & $6.9 \times 10^{17}$ \\
$2198 \mathrm{I}$ & $2.3 \times 10^{17}$ & 5.2 & 0.0041 & $9.1 \times 10^{17}$ \\
$2198 \mathrm{II}$ & $6.3 \times 10^{17}$ & 6.5 & 0.0073 & $1.6 \times 10^{18}$ \\
$2193 \mathrm{I}$ & $8.9 \times 10^{17}$ & only $\left(d^{5}+\mathrm{h}\right)$ & 0.026 & $5.8 \times 10^{18}$ \\
$2193 \mathrm{II}$ & $2.8 \times 10^{18}$ & only $\left(d^{5}+\mathrm{h}\right)$ & 0.049 & $1.1 \times 10^{19}$
\end{tabular}

The ratio of the areas under $\mathrm{A}^{0}$ lines to $\mathrm{A}^{-}$lines differed from sample to sample and it increased with Mn concentration. Corresponding values are given in the Table and they were used in fitting the observed magnetization dependence on magnetic field (see below).

The results of GaAs:Mn magnetization measurements performed at $2 \mathrm{~K}$, corrected for diamagnetic contribution of pure GaAs and plotted as a function of magnetic field, are shown in Fig. 2. In general, overall paramagnetic (PM) behavior was observed. At high temperatures magnetization showed a linear magnetic field dependence. At low temperatures, a pronounced tendency to saturation was observed. Any significant difference between magnetization of the same sample measured at the three different orientations of magnetic field $(\boldsymbol{B}\|[100], \boldsymbol{B}\|[110], \boldsymbol{B} \|[111])$ was not found. In other words, no magnetic anisotropy was observed for GaAs:Mn bulk samples. Such behavior suggested spherically symmetrical configuration of $\mathrm{Mn}$ ion. The experimental data in case of low Mn concentration $\left(p=3 \times 10^{16} \mathrm{~cm}^{-3}\right)$ could be described by the classical Brillouin function with $S=5 / 2$. On the other hand, for samples with a higher Mn content, magnetization should be described by the presence of two types of centers: $\mathrm{Mn}^{2+}\left(d^{5}\right)$ and $\mathrm{Mn}^{2+}\left(d^{5}\right)+\mathrm{h}[6]$.

In Fig. 2 solid lines represent fits to the experimental data. In calculations, the assumed ratio of concentration of neutral acceptor $\mathrm{Mn}^{2+}\left(d^{5}\right)+\mathrm{h}$ to singly ionized acceptor $\mathrm{Mn}^{2+}\left(d^{5}\right)$ was the one estimated from ESR measurements for each sample. For the samples \#2193 I and II the presence of only neutral acceptor $\mathrm{Mn}^{2+}\left(d^{5}\right)+\mathrm{h}$ was assumed. The only fitting parameter was the total concentration of Mn centers, which has been shown next to the fitting curves (Fig. 2).

Hall measurements confirmed the expected $p$-type conductivity of GaAs:Mn samples at room temperature (RT) and the measured RT hole concentration was in the range of $3 \times 10^{16}$ to $3 \times 10^{18} \mathrm{~cm}^{-3}$. Hall concentration, mobility, and resistivity 

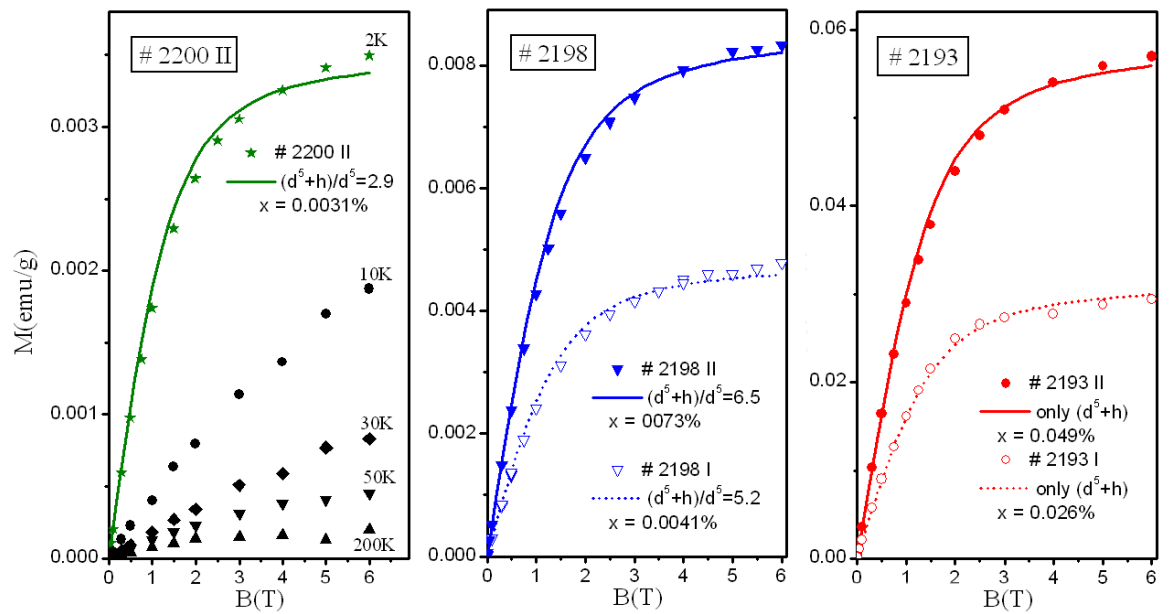

Fig. 2. Magnetization of GaAs:Mn samples as a function of magnetic field, measured at $2 \mathrm{~K}$. Solid lines present fits to the experimental data, assuming the ratios of neutral acceptor $\left(d^{5}\right)$ concentration to singly ionized $\left(d^{5}+\mathrm{h}\right)$ as estimated from ESR measurements. For each sample, this ratio together with the obtained total Mn concentration is shown next to the fitting curves.
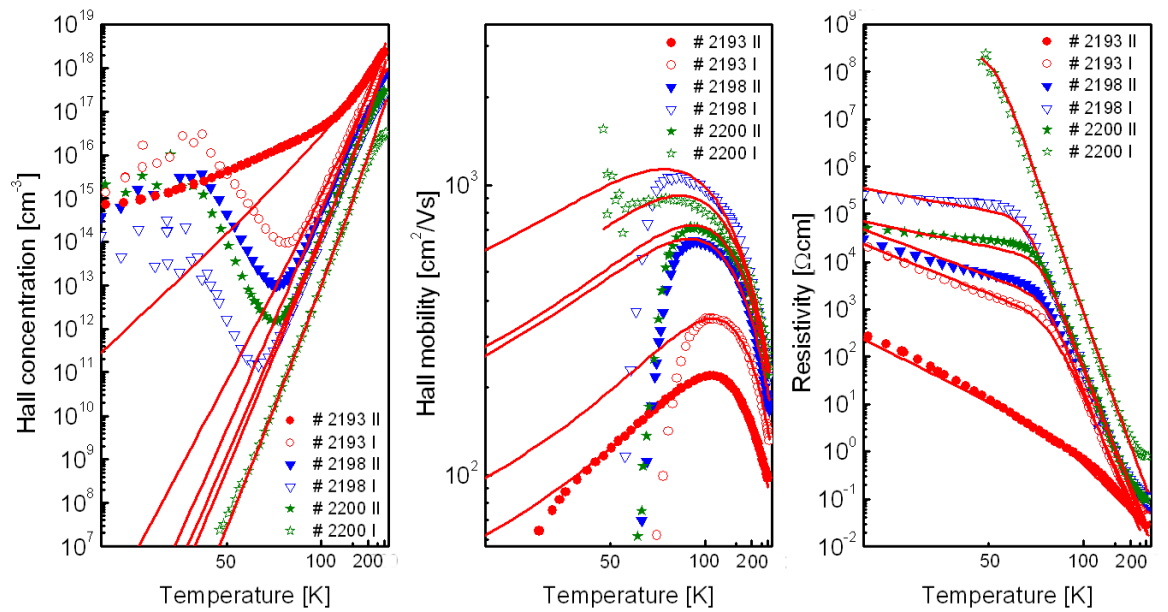

Fig. 3. Hall concentration, mobility, and resistivity measured for GaAs:Mn samples with different Mn concentrations. Solid lines show theoretical calculations using the model presented in [6].

as a function of temperature for the studied samples are shown in Fig. 3. Two mechanisms of conductivity were revealed. Valence band transport dominated above $70 \mathrm{~K}$, whereas hopping conductivity within Mn impurity band was present at lower temperature, and its contribution extended over a wider temperature range with an increase in Mn content. From the analysis of the hopping conductivity, 
taking into account Shklovskii model [7], the effective radius of Mn acceptor in GaAs was estimated as $a=11 \pm 3 \AA$. This value is the highest from all the series of $\mathrm{Mn}$ doped III-V compounds studied by us previously (GaN, GaP, and InP) [8].

\section{Summary}

ESR studies showed the existence of two charge states of Mn acceptor in bulk GaAs:Mn crystals: singly ionized $\mathrm{A}^{-}, \mathrm{Mn}^{2+}\left(d^{5}\right)$, and neutral $\mathrm{A}^{0}, \mathrm{Mn}^{2+}\left(d^{5}\right)+$ hole. Experimental data of magnetization measured as a function of magnetic field were described taking into account these two types of centers. In calculations, the assumed ratio of concentration of neutral acceptor $\mathrm{A}^{0}$ to singly ionized acceptor $\mathrm{A}^{-}$ was taken from corresponding ESR line intensities. Total Mn concentrations in the measured samples were obtained as the only fitting parameter. These values were used in modeling of electrical transport of GaAs:Mn samples, in which valence band transport dominated above $70 \mathrm{~K}$ and hopping conductivity within Mn impurity band at lower temperatures. From the analysis of the hopping conductivity, the effective radius of Mn acceptor in GaAs was estimated as $a=11 \pm 3 \AA$.

\section{Acknowledgments}

This work was supported by the grant No. PBZ-KBN-044/P03/2001 from the State Committee for Scientific Research (Poland).

\section{References}

[1] R.A. Chapman, W.G. Hutchinson, Phys. Rev. Lett. 18, 443 (1967).

[2] N. Almeleh, B. Goldstein, Phys. Rev. 128, 1568 (1962); R. Bleekrode, J. Dieleman, H.J. Vegter, Phys. Lett. 2, 355 (1962).

[3] J. Schneider, U. Kufmann, W. Wilkening, M. Baeumler, Phys. Rev. Lett. 59, 240 (1987); V.F. Masterov, K.F. Sztelmach, M.H. Barbaszov, Fiz. Tekh. Poluprovodn. 22, 654 (1988); N.P. Baran, V.M. Maksimenko, Yu.G. Semenov, V.Ya. Bratus, A.V. Markov, JETP Lett. 55, 101 (1992).

[4] D.C. Look, Semicond. Semimet. 38, 91 (1993).

[5] V.F. Masterov, S.B. Mikhrin, B.E. Samotukov, K.F. Shtel'makh, Sov. Phys. Semicond. 19, 1291 (1985); G.G.P. van Gorkom, A.T. Vink, Solid State Commun. 11, 767 (1972).

[6] Th. Frey, M. Maier, J. Schneider, M. Gehrke, J. Phys. C, Solid State Phys. 21, 5539 (1988).

[7] B.I. Shklovskii, Fiz. Tekh. Poluprov. 6, 1197 (1972).

[8] A. Wołoś, Ph.D. thesis, Warsaw University, Physics Department, Warsaw 2004, ch. 6 . 